\title{
Signal manipulation with a PT-symmetric coupler embedded into an array of optical waveguides
}

\author{
S. V. Suchkov ${ }^{1 \dagger}$, D. I. Borisov ${ }^{2}$, A. A. Sukhorukov ${ }^{1}$, Yu. S. Kivshar ${ }^{1}$ \\ †sergey.v.suchkov@gmail.com \\ ${ }^{1}$ Nonlinear Physics Centre, Australia National University, ACT 2601, Canberra, Australia \\ ${ }^{2}$ Institute of Mathematics USC, RAS, 112 Chernyshevsky St., 450008 Ufa, Russia
}

\begin{abstract}
We study the scattering of linear waves in a long waveguide array with a parity-time (PT) - symmetric defect created by two waveguides with balanced gain and loss. We present exact solutions for the scattering of linear waves on such a defect. We reveal that the reflected and transmitted linear waves can be amplified substantially after interaction with the PT-symmetric defect thus allowing an active control of the wave propagating through the array.
\end{abstract}

Keywords: PT-symmetry, waveguide, scattering.

\section{Introduction}

Bender and co-authors have developed a possible generalization of quantum theory by noting that a class of non-Hermitian Hamiltonians possesses real spectra under the parity-time (PT) - symmetry condition, where paritytime means spatial reflection and time reversal [1,2]. This mathematical discovery has initiated numerous theoretical and experimental studies of open systems with balanced gain and loss in optical [3-19], electrical [20,21] and mechanical [22-25] systems.

Photonic structures composed of coupled waveguides with loss and gain regions offer new possibilities for shaping optical beams and pulses compared to conservative structures [3-6]. Such structures can be designed as optical analogues of complex PT-symmetric potentials, which can have a real spectrum corresponding to the conservation of power for optical eigenmodes, however the beam dynamics can demonstrate unique features distinct from conservative systems due to nontrivial wave interference and phase transition effects [7-12]. Recently, PT-symmetric properties in optical couplers composed of two waveguides were demonstrated experimentally [13,14]. Various schemes have been suggested to tailor beam shaping and switching using PT - symmetric structures, including introduction of fabricated defects in periodic lattices [15] and employing self-induced refractive index change in nonlinear structures [1,16-18]. The possibility of a Hamiltonian reformulation of the standard PT-symmetric dimer has been recently demonstrated [26], and it calls for a broader question of the potential Hamiltonian/Hermitian nature of such systems in suitable modified variables.

In this paper, we suggest and analyse PT-symmetric systems, where a pair of waveguides with balanced gain and loss is embedded into an array of lossless waveguides, as illustrated schematically in Fig.1. Since the active region is confined at only two waveguides, it can be anticipated that the experimental realization of such a structure can be simpler compared to the previously considered cases of nonlinear structures with a periodic arrangement of gain and loss elements [14-16]. On the other hand, the proposed structure offers a full potential for amplification and filtering.

We use the coupled-model equations $[12,14,16]$ to model the beam propagation in a linear waveguide array with a local inhomogeneity created by a pair of PT - symmetric waveguides with balanced gain and loss (see Fig.1):

$$
\begin{aligned}
& i \frac{d a_{j}}{d z}+C_{1} a_{j-1}+C_{1} a_{j+1}=0, j \neq 0,1 \\
& i \frac{d a_{0}}{d z}+i \rho a_{0}+C_{1} a_{-1}+C_{2} a_{1}+\Delta n a_{0}=0, \\
& i \frac{d a_{1}}{d z}-i \rho a_{1}+C_{1} a_{2}+C_{2} a_{0}+\Delta n a_{1}=0
\end{aligned}
$$

where $j$ is the waveguide number, $z$ is the propagation distance, $a_{j}$ are the mode amplitudes at waveguides, $\rho>0$ defines the rate of loss at zeroth and gain at first waveguide,

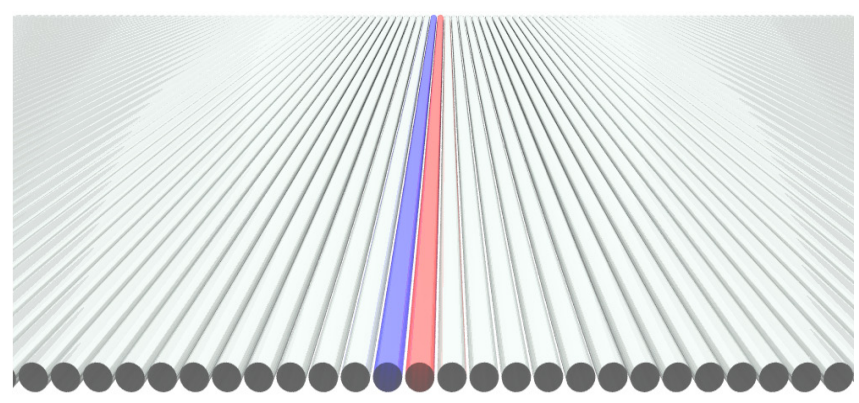

Fig. 1. Schematic of an optical waveguide array with a pair of PT-symmetric waveguides at the sites with balanced gain and loss. Blue colour represents loss, while red colour - gain. 
and $C_{1,2}$ are the coupling coefficients between the modes of waveguides that can be tuned by changing the distance between the waveguides. We introduce here parameter $\Delta n$, which defines the difference of the refractive indexes of the PT-coupler and the other conservative waveguides.

\section{Wave Scattering}

We start with the analysis of propagation of waves in the array, which does not include the PT-symmetric coupler. Thus, for $C_{1}=C_{2}, \Delta n=0$, and $\rho=0$ the waveguide array supports the eigenmode solutions in the form of FloquetBloch modes, $a_{j}(z)=A \exp [i k j-i \beta z]$, where $A$ is an amplitude, $k$ is Bloch wave number, and $\beta$ is a propagation constant obeying the following diffraction relation:

$$
\beta=-2 C_{1} \cos k \text {. }
$$

In a general case, the presence of gain and loss at zeroth and first waveguides and the difference of the coupling constant and refractive index between these two waveguides from the other ones play the role of scatterer of the linear waves. In order to calculate the transmission and reflection coefficients we consider solution to Eq.(1) of the form:

$$
\begin{aligned}
& a_{j}=e^{i(k j-\beta z)}+R e^{i(-k j-\beta z)}, j \leq 0, \\
& a_{j}=T e^{i(k j-\beta z)}, j \geq 1 .
\end{aligned}
$$

where the first line represents the incident and the reflected waves, and the second one - the transmitted wave. Substituting Eq.(5) into Eq.(1) one finds the reflection and transmission coefficients.

$$
\begin{aligned}
& T=-\frac{2 i \tilde{C}_{1} e^{-i k} \sin k}{D}, \\
& R=\frac{1-\tilde{C}_{1}^{2}-\Delta \tilde{n}^{2}-\tilde{\rho}^{2}+2 \tilde{C}_{1} \Delta \tilde{n} \cos k+2 \tilde{C}_{1} \tilde{\rho} \sin k}{D}
\end{aligned}
$$
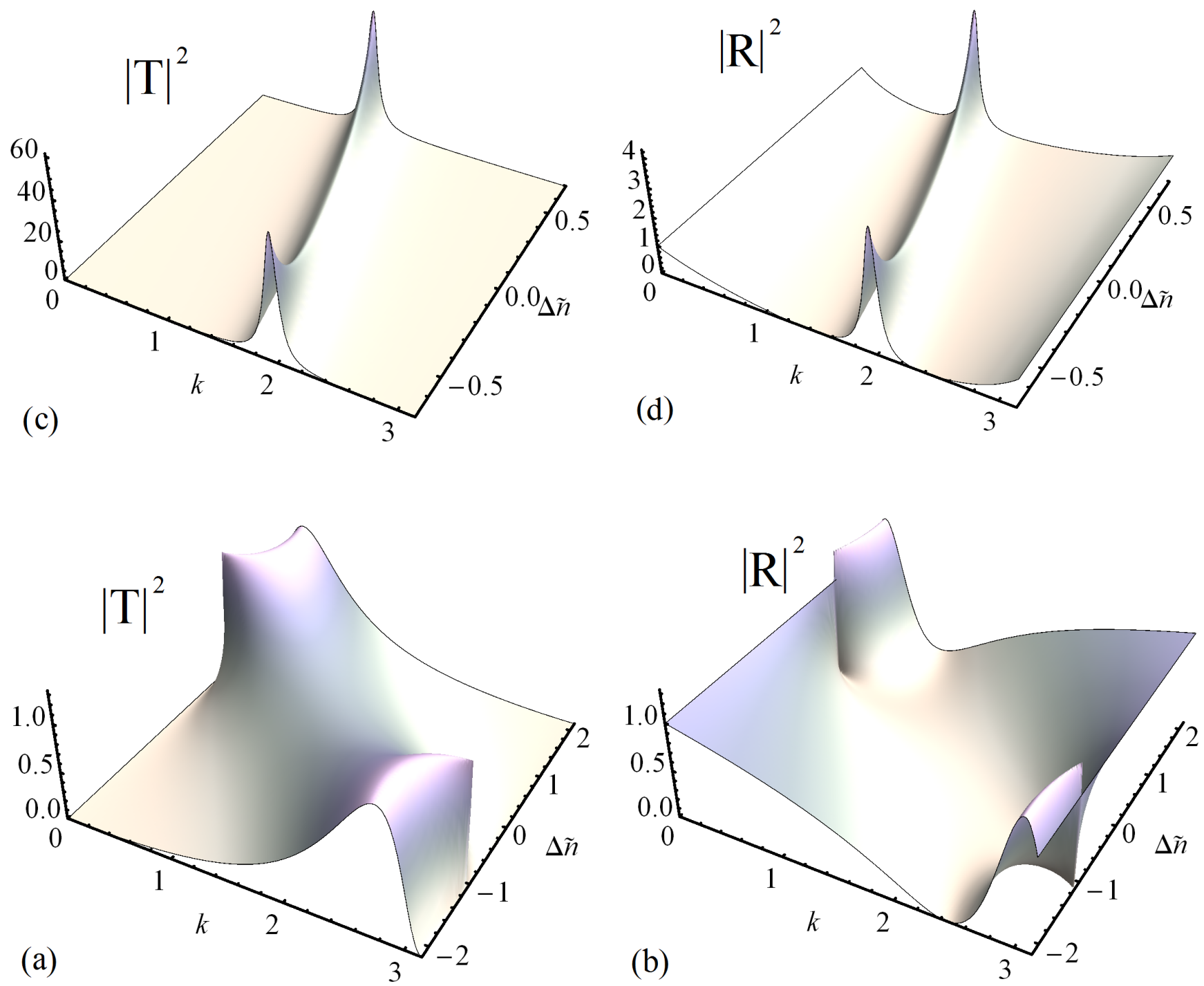

Fig. 2. Scattering coefficients $|T|^{2}$ and $|R|^{2}$ as the functions of the incident wave wavenumber $k$ and the related refractive index $\Delta \tilde{n}$ for two typical cases defined by Eq.(7). In panels (a) and (b) the transmission and reflection coefficients are presented for the model parameters $\tilde{p}=0.5$ and $\tilde{C}_{1}=2$ when the PT-symmetry is conserved for any value of $\Delta \tilde{n}$. In panels (c) and (d) the same is shown but for $\tilde{p}^{=}=2$ and $\tilde{C}_{1}=2$. In this case, PT-symmetry is conserved only for $|\Delta \tilde{n}|<1$. 
where

$$
\begin{aligned}
& D=\left(\Delta \tilde{n}-\tilde{C}_{1} e^{-i k}\right)^{2}+\tilde{\rho}^{2}-1 \\
& \tilde{C}_{1}=C_{1} / C_{2}, \tilde{\rho}=\rho / C_{2} \text { and } \Delta \tilde{n}=\Delta n / C_{2} .
\end{aligned}
$$

There are several points following from the analysis of Eq.(6): first, the intensity of the transmitted wave does not depend on whether the incident wave hits the waveguide with gain or with loss, while the intensity of the reflected wave does $[T(\rho)=T(-\rho)$ but $R(\rho) \neq R(-\rho)]$. Second, if $T=0$ $(R=0)$ for some parameter values, then we have $R=-1$ $(|T|=1)$. Third, the PT symmetry breaking condition takes the form:

$$
\tilde{\rho}_{c r i t}^{2}=\max \left[1,1+\tilde{C}_{1}^{2}-\Delta \tilde{n}^{2}\right]
$$

This means that if $\tilde{\rho}^{2} \geq \tilde{\rho}_{\text {crit }}^{2}$ then the PT-symmetry is broken and the spectrum of the systems possesses complex eigenvalues. Fourth, there is a maximum of transmission coefficient for some wavenumber $k$, which is defined by the relation:

$$
k=\operatorname{Arccos}\left[\left(\frac{\tilde{C}_{1}^{2}+\Delta \tilde{n}^{2}+\tilde{\rho}^{2}-1}{2 \tilde{C}_{1} \Delta \tilde{n}}\right)^{ \pm 1}\right]
$$

where sign \pm is determined by the domain of the arccosine function. In the next section we present several examples of the scattering coefficients for typical cases of relation between model parameters.

\section{Illustrations}

We now illustrate the transmission and reflection coefficients, $|T|^{2}$ and $|R|^{2}$ respectively, for typical cases which are determined by Eq.(7). These coefficients show which part of the incident wave energy passes through the defect and which part reflects from it. If some of the coefficients exceeds unity then reflected and/or transmitted waves have intensity higher than the intensity of the incident wave. First typical case is $\tilde{\rho}^{2}<1$. At such a value of the gain/loss coefficient PT-symmetry of the system is conserved regardless of other model parameters. In Fig.2a,b the transmission and reflection coefficients are presented for the model parameters $\tilde{\rho}=0.5$ and $\tilde{C}_{1}=2$ It is seen that for some value of wavenumber $k$ and $\Delta \tilde{n}$ both reflected and transmitted waves can be amplified by PT-symmetric defect. The second case corresponds to gain/loss coefficient limited by the constrain parameter $1 \leq \tilde{\rho}^{2}<1+\tilde{C}_{1}{ }^{2}-\Delta \tilde{n}^{2}$. In Fig. $2 \mathrm{c}, \mathrm{d}$ we present the scattering coefficients for the following parameter values $\tilde{\rho}=2$ and $\tilde{C}_{1}=2$. It is remarkable that the transmission and reflection coefficients have a strong, narrow resonance around the wavenumber determined by Eq.(8). It means that PT-symmetric coupler at such model parameters can be used for amplification and filtering of input signal. It gives a wide prospect of employing PT-symmetric optical structures for the light beams manipulation.

\section{Conclusion}

We have proposed a discrete model describing the propagation of electromagnetic waves in an array of optical waveguides with a two-site defect with balanced gain and loss created by a pair of PT-symmetric waveguides. Our model differs from the previously considered models by an additional parameter $\Delta n$, which defines a difference of the refractive indices of the PT-coupler and other conservative waveguides. In the case of a long array (when the effects of boundaries are neglected), we have derived exact relations for the transmission and reflection coefficients of linear waves scattered by the PT-symmetric defect. We have found that the reflected and transmitted linear waves can be substantially amplified by the PT-symmetric defect. Due to the presence of a narrow resonance behaviour of the scattering coefficients, the considered structure can be used for an effective filtering of input signals. Our results demonstrate that a pair of PT-symmetric waveguides with balanced gain and loss can provide a flexible and active control of the propagation of optical beams in waveguide arrays.

S.V.S. thanks Dynasty Foundation for the financial support. D.I.B. was supported by a grant of the Russian Science Foundation (project no. 14-11-00078).

\section{References}

1. C.M. Bender, S. Boettcher. Phys. Rev. Lett. 80, 5243 (1998).

2. C. M. Bender, D. C. Brody, H. F. Jones. Phys. Rev. Lett. 89, 270401 (2002).

3. Y.J. Chen, A. W. Snyder, D. N. Payne. IEEE J. Quantum Electron. 28, 239 (1992).

4. A. Ruschhaupt, F. Delgado, J. G. Muga. J. Phys. A38, L171 (2005).

5. R. El-Ganainy, K.G. Makris, D. N. Christodoulides, Z.H. Musslimani. Opt. Lett. 32, 2632 (2007).

6. S. Klaiman, U. Gunther, and N. Moiseyev. Phys. Rev. Lett. 101, 080402 (2008).

7. K.G. Makris, R. El-Ganainy, D.N. Christodoulides, Z.H. Musslimani. Phys. Rev. Lett. 100, 103904 (2008).

8. M. V. Berry. J. Phys. A41, 244007 (2008).

9. S. Longhi. Phys. Rev. Lett. 103, 123601 (2009).

10. S. Longhi. Phys. Rev. A81, 022102 (2010).

11. K.G. Makris, R. El-Ganainy, D. N. Christodoulides, Z.H. Musslimani. Phys. Rev. A81, 063807 (2010).

12. M.C. Zheng, D. N. Christodoulides, R. Fleischmann, T. Kottos. Phys. Rev. A82, 010103 (2010).

13. A. Guo, G. J. Salamo, D. Duchesne, R. Morandotti, M. Volatier-Ravat, V. Aimez, G.A. Siviloglou, D. N. Christodoulides. Phys. Rev. Lett. 103, 093902 (2009).

14. C.E. Ruter, K.G. Makris, R.El-Ganainy, D.N. Christodoulides, M. Segev, D. Kip. Nature Physics. 6, 192 (2010).

15. K. Y. Zhou, Z. Y. Guo, J. C. Wang, and S. T. Liu. Opt. Lett. 35, 2928 (2010). 
16. Z.H. Musslimani, K.G. Makris, R. El-Ganainy, D. N. Christodoulides. Phys. Rev. Lett. 100, 030402 (2008).

17. Z.H. Musslimani, K.G. Makris, R. El-Ganainy, D. N. Christodoulides. J. Phys. A41, 244019 (2008).

18. S. V. Dmitriev, A. A. Sukhorukov, Yu. S. Kivshar. Opt. Lett. 35, 2976 (2010).

19. H. Ramezani, T. Kottos, R. El Ganainy, D. N. Christodoulides. Phys. Rev. A82, 043803 (2010).

20. A. A. Sukhorukov, Z. Xu, Yu. S. Kivshar. Phys. Rev. A82, 043818 (2010).
21. J. Schindler, A. Li, M.C. Zheng, F.M. Ellis, T. Kottos. Phys. Rev. A84, 040101 (2011).

22. J. Schindler, Z. Lin, J.M. Lee, H. Ramezani, F.M. Ellis T. Kottos, J. Phys. A. Math. Theor. 45, 444029 (2012).

23. C.M. Bender, B. Berntson, D. Parker, E. Samuel. Am. J. Phys. 81, 173 (2013).

24. P. G. Kevrekidis. Phys. Rev. A89, 010102 (2014).

25. Saxena, and A. Stefanov. Phys. Rev. E88, 023203 (2013).

26. D. Saadatmand, S.V. Dmitriev, D.I. Borisov, P. G. Kevrekidis. Phys. Rev. E90, 052902 (2014).

27. I. V. Barashenkov. Phys. Rev. A90, 045802 (2014). 